\title{
.
}

RESEARCH

\section{Genetic predisposition to neural crest-derived tumors: revisiting the role of KIF1B}

\author{
Catherine Cardot Bauters ${ }^{1}$, Emmanuelle Leteurtre ${ }^{2}$, Bruno Carnaille ${ }^{3}$, Christine Do Cao ${ }^{1}$, Stéphanie Espiard ${ }^{1}$, \\ Malo Penven ${ }^{4}$, Evelyne Destailleur ${ }^{4}$, Isabelle Szuster ${ }^{4}$, Tonio Lovecchio ${ }^{4}$, Julie Leclerc ${ }^{2,4}$, Fredéric Frénois ${ }^{5}$, \\ Emmanuel Esquivel ${ }^{6}$, Patricia L M Dahia ${ }^{6}$, Emilie Ait-Yahya ${ }^{7}$, Michel Crépin ${ }^{4}$ and Pascal Pigny ${ }^{4}$
}

${ }^{1} \mathrm{CHU}$ Lille, Service d'Endocrinologie, Diabétologie, Métabolisme-Nutrition, Hôpital Claude Huriez, Lille, France

2Univ. Lille, Inserm, CHU Lille, UMR-S 1277-CANTHER, Cancer Heterogeneity, Plasticity \& Resistance to Therapies, Lille, France

${ }^{3} \mathrm{CHU}$ Lille, Service de Chirurgie Endocrine, Hôpital Claude Huriez, Lille, France

${ }^{4} \mathrm{CHU}$ Lille, Service de Biochimie Hormonologie, Métabolisme, Nutrition-Oncologie, Centre de Biologie Pathologie Génétique, Lille, France

5Univ. Lille, CHU Lille, EA-7364 RADEME, Faculté de Médecine, Lille, France

${ }^{6}$ Dept Medicine, Mays Cancer Center, University of Texas Health Science Center at San Antonio, San Antonio, Texas, USA

${ }^{7} \mathrm{CHU}$ Lille, Institut de Biochimie \& Biologie Moléculaire, Centre de Biologie Pathologie Génétique, Lille, France

Correspondence should be addressed to P Pigny: pascal.pigny@chru-lille.fr

\begin{abstract}
Objective: We previously described a family in which predisposition to pheochromocytoma (PCC) segregates with a germline heterozygous KIF1B nucleotide variant (c.4442G>A, p.Ser1481Asn) in three generations. During the clinical follow-up, one proband's brother, negative for the KIF1B nucleotide variant, developed a bilateral PCC at 31 years. This prompted us to reconsider the genetic analysis.

Design and methods: Germline DNA was analyzed by next-generation sequencing (NGS) using a multi-gene panel plus MLPA or by whole exome sequencing (WES). Tumorderived DNA was analyzed by SnapShot, Sanger sequencing or NGS to identify loss-ofheterozygosity ( $\mathrm{LOH}$ ) or additional somatic mutations.

Results: A germline heterozygous variant of unknown significance in MAX (c.145T>C, p.Ser49Pro) was identified in the proband's brother. Loss of the wild-type MAX allele occurred in his PCCs thus demonstrating that this variant was responsible for the bilateral PCC in this patient. The proband and her affected grandfather also carried the MAX variant but no second hit could be found at the somatic level. No other pathogenic mutations were detected in 36 genes predisposing to familial PCC/PGL or familial cancers by WES of the proband germline. Germline variants detected in other genes, TFAP2E and TMEM214, may contribute to the multiple tumors of the proband.

Conclusion: In this family, the heritability of PCC is linked to the MAX germline variant and not to the KIF1B germline variant which, however, may have contributed to the occurrence of neuroblastoma (NB) in the proband.
\end{abstract}

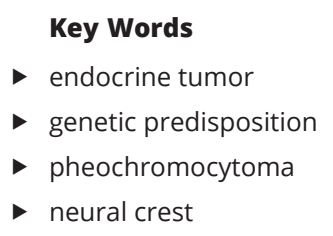

Endocrine Connections (2020) 9, 1042-1050

\section{Introduction}

We previously described a family in which predisposition to pheochromocytoma (PCC), segregates into three generations with a germline heterozygous nucleotide variant of KIF1B (c.4442G>A, p.Ser1481Asn) which encodes the kinesin-like protein KIF1B (1). KIF1B isoform $\beta(\mathrm{KIF} 1 \mathrm{~B} \beta)$ is a molecular motor protein that participates in the transport of synaptic vesicle precursors and is essential for neuronal survival and differentiation (2). In vitro, the p.Ser1481Asn variant decreases the ability of KIF1B $\beta$ to promote the apoptosis of primary rat sympathetic neurons (3) and thus may facilitate tumorigenesis later on. Conversely enforced expression of KIF1B resulted in 


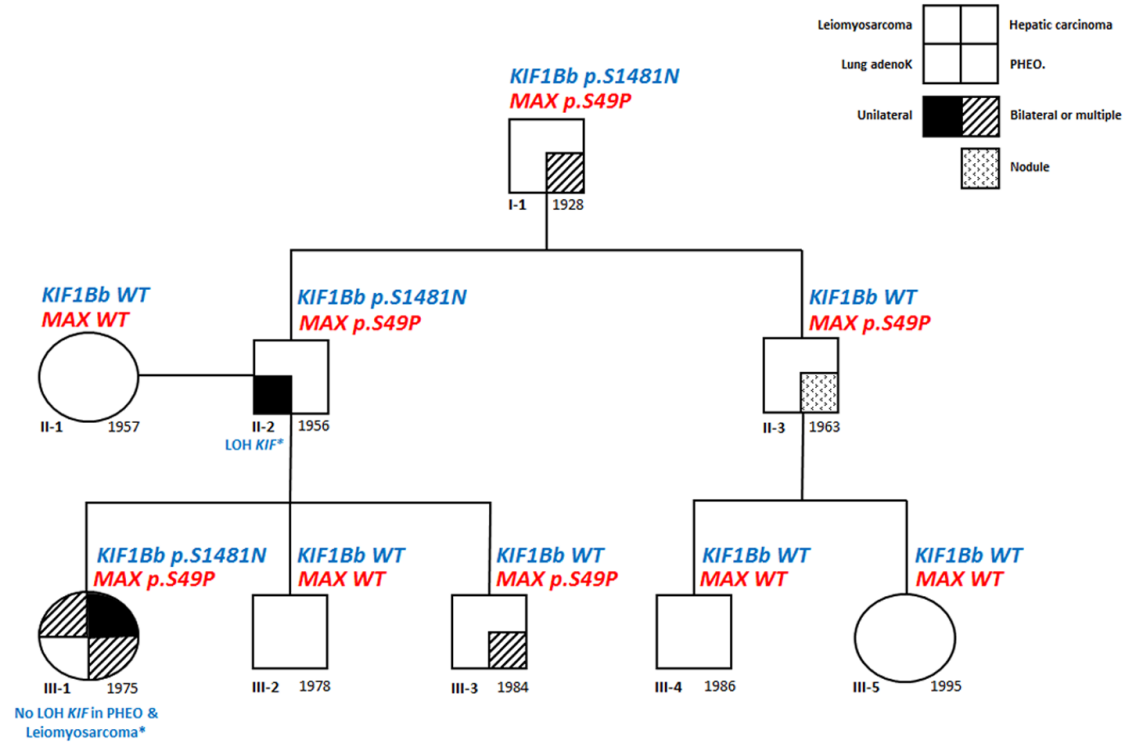

Figure 1

Phenotype and genotype of the family. The proband is patient III.1. an induction of apoptosis of neuroblastoma (NB) cells (4). Thus, the KIF1B $\beta$ neuronal pro-apoptotic effect combined with the mapping of $K I F 1 B$ on chromosome $1 \mathrm{p} 36$, a region frequently deleted in PCC and NB (5), suggested that $K I F 1 B$ might function as a tumor suppressor gene (TSG) in these diseases (3). However, in our kindred, we did not identify a loss of the wild type (WT) allele of KIF1B at the somatic level in the PCC or NB of the proband, deviating from the Knudson two hits theory (1). At that time, we thus hypothesized that the p.S1481N variant of KIF1B $\beta$ functions in haploinsufficiency in these tumors (1) but its exact mechanisms of action remain unclear (6).

Since our initial report, the large size KIF1B gene has been rarely incorporated in the PCC/PGL gene panels which are analyzed in patients with PCC or PGL by next-generation sequencing to identify familial tumors. Welander et al. (7) described one PCC patient with a germline variant of $K I F 1 B$ classified as disease-causing in their cohort thus representing a prevalence of $1.1 \%$. The PCC had a sporadic presentation in this woman who later presented with endometrial carcinoma. CurrasFreixes et al. (8) reported three germline variants of unknown significance (VUS) of KIF1B in their cohort of PCC patients, representing a prevalence of $0.66 \%(3 / 453)$ in line with our own estimation of the prevalence of KIF $1 B$ VUS at $1.3 \%$ (1/74 patients with PCC/PGL analyzed by NGS between 2017 and 2019, unpublished data).

In conclusion, only $1-2 \%$ of the patients with PCC or PGL had a germline pathogenic variant of KIF1B thus leading to frequent questioning on its involvement in the heritability of PCC/PGL $(9,10)$.
At the time of our report in 2008, the proband's two brothers, who did not carry the KIF1B germline variant, were clinically asymptomatic (1). However, during the clinical follow-up, one of proband's brother developed a bilateral PCC at 31 years. This prompts us to extend the genetic analysis in this family and to reconsider the molecular pathogenesis of the PCC.

\section{Materials and methods}

\section{Clinical data}

The pedigree of the family has been updated in Fig. 1. In brief, the proband III-1 developed NB of the broad ligament at 17 months which was treated by surgery, radiation therapy and chemotherapy. At age 22 years, she developed a right PCC plus a ganglioneuroma at the site of the original NB and also an ileal schwannoma. At that time, she had hypertension and high normetanephrines levels (exact data not available). Six years later, the patient underwent adrenal surgery for a left PCC associated with a mature ganglioneuroma. At the same time, a well-differentiated leiomyosarcoma arising from the mesosigmoid was detected and surgically removed. At 39 years, several cutaneous metastases of the leiomyosarcoma were surgically removed. At 42 years, a 9-cm moderately differentiated hepatic carcinoma was diagnosed and surgically removed. Finally, at 43 years a uterine leiomyoma, and two metastases (parietal and peritoneal) from the leiomyosarcoma were removed. Her paternal grandfather (I-1) had bilateral PCC at 70 years (c) 2020 The authors Published by Bioscientifica Ltd

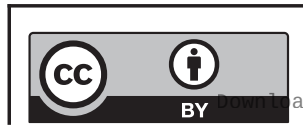

This work is licensed under a Creative Commons Attribution 4.0 International License. ded from Bioscientifica.com at 04/26/2023 12:58:44AM 
and her father (II-2) had a lung adenocarcinoma at 47 years and prostatic cancer at 54 years. The proband's youngest brother (III-3) presented at 31 years with a cardiomyopathy complicated by a Takotsubo's syndrome which led to the diagnosis of bilateral PCC. The proband's paternal uncle (II-3) was diagnosed at 56 years with an oligo-symptomatic adrenal nodule exhibiting a high $\left[{ }^{18} \mathrm{~F}\right]$-DOPA uptake at PET.

\section{Custom endocrine tumors NGS panel}

In compliance with the French regulation, each patient gave his/her written informed consent before performing genetic testing which is an integral part of the patient's care. Moreover, this protocol was reviewed and validated by the Ethical Committee (Comité de Protection des Personnes Nord Ouest IV) under the number HP 20/04. A custom panel based on the hybridization and capture technology (Haloplex, Agilent Technologies) was designed to be compatible with the Illumina MiSeq platform. Probes covered the coding regions and intronic flanking sites of 21 susceptibility genes for endocrine tumors, either PCC/PGL (VHL, RET, SDHA, SDHAF2, SDHB, SDHC, SDHD, TMEM127, KIF1B, MAX, PHD2) or pituitary tumors/hyperparathyroidism (MEN1, HRPT2, AIP, CASR, CYP24A1, GCM2, PTH, GNA11, AP2S1, CDKN1B). Genomic DNA extracted from blood cells was fragmented with restriction enzymes; then digested DNA was hybridized to Haloplex probes which resulted in circularization of DNA fragments and sample indexing. Target DNA was captured with streptavidin-coated magnetic beads, ligated and eluted before bridge PCR amplification of the libraries. After quantification of enriched target DNA, samples were pooled for multiplexed sequencing. NGS sequencing data were aligned to hg19 human reference and annotated using two independent bioinformatic pipelines (alignment using bwa v0.7.15-r1140 followed by best practices for germline variant detection using GATK v3.7 (11) and SeqNext V4.4 (JSI) (12)). Data were filtered using an in-house database (DVD) to remove recurrent sequencing errors. Regions with coverage $<30 \mathrm{X}$ were reanalyzed using Sanger sequencing.

\section{Somatic cancer panel analysis}

DNA extracted from three separate regions of the frozen left PCC from proband (III-1) was analyzed for somatic nucleotide variants in hot spot regions of 48 commonly mutated cancer genes using the Illumina TruSeq Amplicon Cancer Panel (ABL1, AKT1, ALK, APC, ATM, BRAF, CDH1, CDKN2A, CSF1R, CTNNB1, EGFR, ERBB2, ERBB4, FBXW7,
FGFR1, FGFR2, FGFR3, FLT3, GNA11, GNAQ, GNAS, HNF1A, HRAS, IDH1, JAK2, JAK3, KDR, KIT, KRAS, MET, MLH1, MPL, NOTCH1, NPM1, NRAS, PDGFRA, PIK3CA, PTEN, PTPN11, RB1, RET, SMAD4, SMARCB1, SMO, SRC, STK11, TP53, VHL), run on Illumina MiSeq following standard protocols, and analyzed using the filter settings described previously.

\section{WES (whole exome sequencing) analysis}

Genomic DNA extracted from fresh blood cells from the proband and her father with QiAmp DNA mini kit (Qiagen) was captured using Agilent in-solution enrichment methodology with their biotinylated oligonucleotides probes library (SureSelect Clinical Research Exome V2, Agilent Technologies), followed by paired-end 75 bases massively parallel sequencing on Illumina HiSeq4000 (IntegraGen SA, Evry, France), as reported (13). Sequence capture, enrichment and elution were performed according to manufacturer's instruction and protocols (SureSelect, Agilent) without modification, except for library preparation performed with NEBNext巴 Ultra kit (New England Biolabs). For library preparation, 600 ng of each genomic DNA were fragmented by sonication and purified to yield fragments of 150-200 bp. Pairedend adaptor oligonucleotides were ligated on repaired fragments then purified and enriched by 8 PCR cycles. A total of 1200ng of the purified Libraries were then hybridized to the SureSelect oligo probe capture library for $72 \mathrm{~h}$. After hybridization and washing, the eluted fraction was PCR-amplified, purified and quantified by qPCR. Each eluted-enriched DNA library was then sequenced on an Illumina HiSeq4000 as paired-end 75 bp reads. Image analysis and base calling were performed using Illumina Real Time Analysis (2.7.7) with default parameters.

\section{Bioinformatic analysis}

After demultiplexing andFASTQgeneration, the paired-end reads were trimmed using TrimGalore v0.4.4. The pairedend reads were then aligned to hg19 human reference genome with BWA v0.7.15-r1140 (11). We applied the GATK v3.7 pipeline (12) for indel realignment, duplicate removal, and performed SNP and INDEL discovery as well as share genotype across both the proband and her father's samples simultaneously according to GATK Best Practices recommendations $(14,15)$. Variants were annotated and filtered with Agilent Technologies Bench Lab NGS v5.0.2. To select putative pathogenic variants, filters were applied as follows (Supplementary Fig. 1, see section on

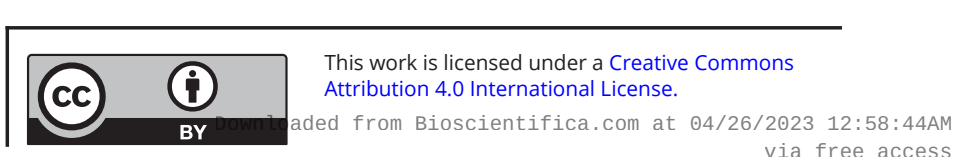


supplementary materials given at the end of this article): (i) variants common to proband and her father were filtered out; (ii) low quality reads were filtered out; (iii) only heterozygous variants were retained; (iv) variants predicted as pathogenic (Supplementary Fig. 1) were retained; (v) variants in exonic regions or near splicing sites were retained; (vi) variants with a frequency $\geq 0.01$ in the general population (using Exac database, release 0.3, 1000 Genomes Phase1 release v3.20101123, ESP6500SI-V2, 1000 Genomes Phase 3 release v5.20130502) were filtered out. Relevant nucleotide variants were validated by Sanger sequencing on Applied Biosystems 3730 platform.

\section{Search for large rearrangements}

The analysis of large rearrangements was performed with the Multiplex Ligation-dependent Probe Amplification (MLPA) technology for $F H$ and $S D H x$ genes and by multiplex PCR (QMPSF) for VHL. MLPA probes (ref. SALSA MLPA Probemix P198, P226 and P429) and reagents were manufactured and supplied by MRC-Holland (Amsterdam, The Netherlands). The MLPA procedure was conducted according to manufacturer's specifications. The amplified probes were analyzed on a 3130XL DNA Analyzer (Life Tech, ThermoFisher). Regarding FH analysis, the proband DNA sample was tested twice in the same run along with 10 negative samples and one positive control with total deletion of the $F H$ gene. Data were interpreted with Coffalyser software (MRC Holland).

\section{LOH analysis}

DNA from formalin-fixed paraffin-embedded (FFPE) tumor samples was extracted using the QIAAmp DNA FFPE kit (Qiagen). Tissue samples of the proband available for $\mathrm{LOH}$ analysis are detailed in Supplementary Table 1. LOH of selected nucleotide variant was searched by SNaPshot analysis as previously described (16). PCR and extension primers details are available upon request. Extension products were analyzed on Applied Biosystems 3730 along with GeneScan 120LIZ molecular marker using the Genemapper software. In addition, visualization of the variant peaks on Sanger sequence traces was done using the Mutation Quantifier tool (Surveyor program, Softgenetics).

\section{Immunohistochemical analysis}

Fumarate Hydratase expression was assessed on paraffinembedded tumors using an anti-FH antibody 1:1000 dilution as previously described (17).

\section{In silico analysis}

Prediction of the missense variant of MAX protein was carried out with the Phyre2 server (Protein Homology/ analogY Recognition Engine V2.0). We compared the deduced human amino-acid 3D structure with the 3D resolved structure of the Homo sapiens MAX protein $(99 \%$ sequence identity) using The PyMOL Molecular Graphics System (v2.0, Schrödinger, LLC). The SuperPose server v.1.0 (18) was used to estimate the structural homology, measuring the average distance between the backbones of superimposed proteins.

\section{Results}

During the clinical follow-up of the family, patient III-3, who did not harbor the KIF1B c.4442G>A nucleotide variant in his germline DNA, developed bilateral PCC at 31 years. His germline DNA was analyzed by NGS using a custom endocrine tumor panel which includes 11 major susceptibility genes for familial PCC/PGL (PHD2, KIF1B $\beta$, SDHA, SDHB, SDHC, SDHD, SDHAF2, VHL, MAX, TMEM127 and RET). The only heterozygous variant identified was in MAX (NM_002382.5): c.145T>C, p.Ser49Pro. A large rearrangement of SDHA, SDHB, SDHC, SDHD, SDHAF2, $F H$ and $V H L$ was excluded by MLPA or QMPSF analysis, respectively. Using the ACMG guidelines and Varsome tool (19), the MAX nucleotide variant was classified as a VUS. The secretory profile of his tumors consisted of increased urinary normetanephrines at $1979 \mathrm{nmol} / \mathrm{mmol}$ of creatinine ( $8 \mathrm{x}$ the upper limit range) with normal total metanephrines at $182 \mathrm{nmol} / \mathrm{mmol}$ of creatinine $(<190)$, reminiscent of the pattern observed in patients with a MAX pathogenic variant (20). The p.Ser49Pro MAX variant is predicted to show the Proline 49 with an opened cycle which is impossible, leading probably to the precipitation of the protein (Fig. 2). Proline is a constrained amino acid due to its pyroxylin cycle hindering the rotation of the $\phi$ angle with the previous amino acid and thus altering the amino acid connection. Therefore, this variant is likely deleterious.

We thus reanalyzed the germline DNAs of the proband (III-1), her father (II-1) and grandfather (I-1), who had been previously found to be carriers of the KIF1B c. $4442 \mathrm{G}>\mathrm{A}$ nucleotide variant (1), using the NGS panel. All also carried the MAX c.145T>C variant at the heterozygous level. No other pathogenic variant in the nine other susceptibility genes for PCC/PGL was identified (Fig. 1). We also excluded a large rearrangement of $S D H A$, $S D H B, S D H C, S D H D, S D H A F 2, F H$ and $V H L$ in the proband

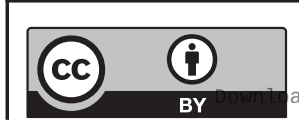

This work is licensed under a Creative Commons Attribution 4.0 International License. ded from Bioscientifica.com at $04 / 26 / 2023$ 12:58:44AM 

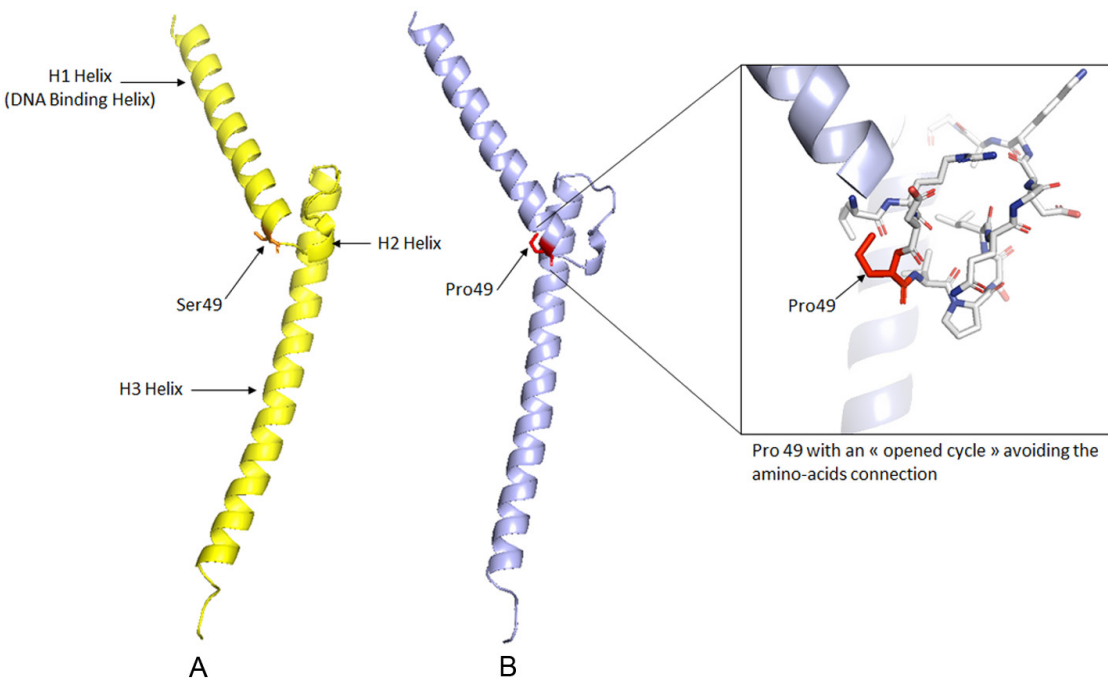

Figure 2

(A) 3D structure of the human native MAX protein (pdb code: 1AN2) (amino acids 22-104); (B) 3D structural prediction of the human Ser49Pro mutated MAX protein (amino acids 22-104). by MLPA or QMPSF analysis, respectively. The MAX c. $145 \mathrm{~T}>\mathrm{C}$ variant was also detected by Sanger sequencing at the heterozygous level in the germline DNA of the proband's paternal uncle (II-3), who was KIF1B negative (Fig. 1). Interestingly, II-3 recently developed, at age 56, a 11-mm hypervascular nodule in his right adrenal which remains clinically silent but strongly uptakes $\left[{ }^{18} \mathrm{~F}\right]$-DOPA during PET, suggestive of PCC or adrenomedullary nodule. Plasma free normetanephrines were at the upper limit of the normal values. Pre-operatively, patient I-1 had high urinary normetanephrines ( $5 \mathrm{x}$ the upper limit range) and chromogranin-A levels (x3 the upper limit range). By contrast, patient II-2, who had a regular follow-up by PET imaging due to his lung and prostatic cancers, never demonstrated an adrenal uptake of $\left[{ }^{18} \mathrm{~F}\right]$-deoxy-glucose. Moreover, his levels of metanepherines/normetanephrines were in the normal range at each follow-up, both elements being in disfavor of a PCC.

Since MAX behaves as a TSG (20), we analyzed the tumor DNAs of patient III-3 and identified loss of the MAX WT allele in both tumors (Fig. 3) suggesting that this variant is indeed responsible for the bilateral PCC in this patient. In contrast, no LOH of the WT allele of MAX was found in the 2 PCCs of the proband (III-1), using DNA obtained from three independent samples, FFPE samples from left and right PCCs (Fig. 3), and one fresh frozen sample from the left PCC (Supplementary Fig. 2). The oldest of the samples (the right PCC) in fact showed complete absence of the variant allele (Fig. 3), which could be due to allelic dropout in the PCR, a well-recognized cause of errors in DNA from suboptimal samples (21). Although the estimated proportion of tumor cells of the left PCC FFPE sample was high (Fig. 3), this estimate was not available from the bulk frozen specimen from this tumor nor the right PCC, so it is unclear to what extent normal cell admixture may have contributed to the allelic count. We also excluded, by Sanger sequencing, the presence of an additional somatic MAX mutation, which might have functioned as the

MAX, NM_002382: c.145t>c

Patient III-3

Blood DNA

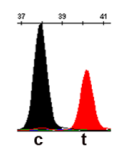

Patient III-1 Blood DNA
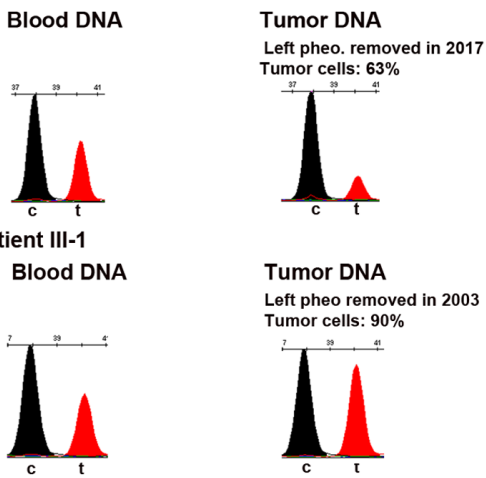

Right pheo removed in 2017 Tumor cells: $63 \%$

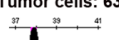

Tumor DNA

Left pheo removed in 2003 Tumor cells: $\mathbf{9 0 \%}$

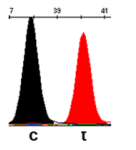

Right pheo removed in 1997

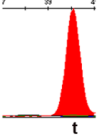

KLHL7, NM_018846: c.1319g>a

Patient III-3

Blood DNA

Tumor DNA

Left pheo removed in 2017 Tumor cells: $63 \%$

Right pheo removed in 2017 Tumor cells: $63 \%$
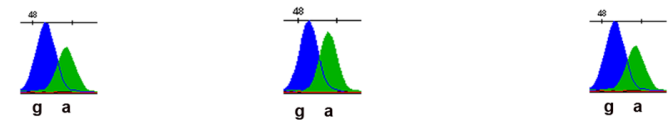

Patient III-1 Blood DNA

Tumor DNA Left pheo removed in 2003
Tumor cells: $90 \%$

Right pheo removed in 1997 ${ }^{49}$

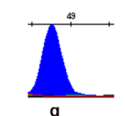

Figure 3

Search for a LOH of MAX and KLHL7 in the DNA extracted from the pheochromocytomas of patients III.1 and III.3 by SnapShot analysis.

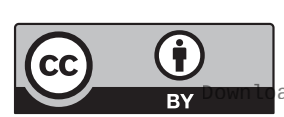

This work is licensed under a Creative Commons Attribution 4.0 International License. 
second hit in the absence of $\mathrm{LOH}$ in the DNA extracted from the left PCC of the proband. We further examined the DNA from the three separate fragments from the left PCC to search for additional somatic mutations in 48 cancer genes using NGS. The three fragments displayed similar variant allele frequencies across these genes, suggesting that the left PCC was homogeneous with respect to both genetic and cell composition. Moreover, these fragments lacked areas of allelic imbalance, in favor of a high level of normal cells in these fragments. Only a few VUS were detected (Supplementary Table 2); however, no variant frequency suggestive of $\mathrm{LOH}$ was observed in these three samples. Finally, FH expression evaluated by immunohistochemistry was conserved on the uterine leiomyoma and pelvic leiomyosarcoma of the proband (Supplementary Fig. 3), which strongly suggests the absence of somatic pathogenic variants of $F H$ responsible for these 2 tumors in the proband.

Since the proband (III-1) and her father (II-2) shared the same genotype for MAX and KIF1B despite very different phenotypes (Fig. 1), we considered that the proband may carry additional variants in known/new susceptibility genes that modified cancer predisposition, which might have been inherited from her mother or have occurred de novo. WES was performed on the germline DNA of III-1 and II-2. First, the proband had no pathogenic variant in NF1, IDH1, IDH2, FH, MDH2 or SLC25A11 which predispose to familial PCC/PGL nor in a group of 30 cancer-predisposing genes (22), that is, APC, ATM, BAP1, BARD1, BMPR1A, BRCA1, BRCA2, BRIP1, CDH1, CDKN2A, CDK4, CHEK2, EPCAM, GREM1, MITF, MLH1, MSH2, MSH6, MUTYH, NBN, PALB2, PMS2, POLD1, POLE, PTEN, RAD51C, RAD51B, SMAD4, STK11 and TP53. Thus, we decided to focus on the nucleotide variants which were present in the germline of III- 1 but absent in II-2 in agreement with our working hypothesis. One hundred twenty-five nucleotide variants were identified as unique to III-1 (Supplementary Fig. 1). Class 1 and 2 variants (benign or probaby benign) were filtered out using Varsome, leading to a list of 24 variants all classified as VUS (Class 3) (Table 1). After the interrogation of several resources such as PUBMED (for a link between the gene of interest and cancer), UniProt (for information on encoded protein function), HGMD (for information on germline mutations currently identified), TCGA (for somatic mutations catalogue), Protein Atlas and CTEX databases (for detailed information on tissue expression), the list was narrowed down to five variants occurring in five genes (Table 1). KLH7 and PKM were good candidate genes for hereditary PCC since they encode proteins expressed in adrenals (23) and are mutated (though rarely) at the somatic level in PCC (TCGA PCPG). RIPK3, TFAP2E and TMEM214 were good candidates for the non-neural crest tumors since they are mutated in sarcomas (TCGA SARC), myomatous neoplasms and in hepatocarcinomas (TCGA LIHC) at the somatic level. No protein expression data were available for TMEM214, TFAP2E and RIPK3 in the Protein Atlas (23). Sanger sequencing confirmed the presence of the five nucleotide variants in the germline of the proband and her mother but absence in her father as expected (Table 2), none were de novo. Patient III-3 was heterozygous only for the KLHL7 variant.

Tumor DNA from eight different FFPE tumors from the proband (Supplementary Table 1) were analyzed by SnapShot to screen for $\mathrm{LOH}$ of the candidate genes. No LOH of KLH7 WT allele was found in the PCC of the proband nor in those of her brother III.3 (Fig. 3). Unfortunately, the data were not informative for $P K M$ (not shown). Regarding TFAP2E, LOH of the WT allele was found in the DNA from the uterine leiomyoma whereas no LOH occurred in the parietal and peritoneal metastasis of the leiomyosarcoma (Fig. 4). By contrast, a LOH of the WT allele of TMEM214 was found in the parietal and peritoneal metastasis of the leiomyosarcoma (Fig. 4) whereas the data were not informative in the hepatocarcinoma (not shown).

\section{Discussion}

The occurrence of a bilateral PCC at a young age in a relative (patient III.3) who did not carry the germline KIF1B c. $4442 \mathrm{G}>\mathrm{A}$ variant suggested that other susceptibility gene(s) might be implicated in the PCC/PGL predisposition of this family. Indeed, analysis of a panel of 11 classic susceptibility genes for PCC/PGL $(17,18)$ by NGS led to the identification of a heterozygous variant of MAX: c.145T>C, p.Ser49Pro. This variant was also found in the germline of the two other individuals with PCC in this family, III-1 and I-1. This variant has not been previously found in the germline of patients with PCC/PGL or at the somatic level based on the LOVD Leiden and TCGA database, respectively. Using the pathogenicity criteria proposed by the $A C M G$, this variant was classified as a VUS rendering the interpretation more complex. However, several elements are in favor of its pathogenic role. First, the secretory pattern of the tumors of patient III-3 and I- 1 is compatible with those classically observed in patients with MAX pathogenic variants (20). Secondly, the loss of the WT allele of MAX in both PCCs of III-3 suggests the role

This work is licensed under a Creative Commons Attribution 4.0 International License. ded from Bioscientifica.com at $04 / 26 / 2023$ 12:58:44AM 


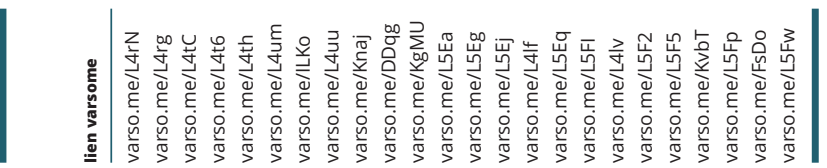

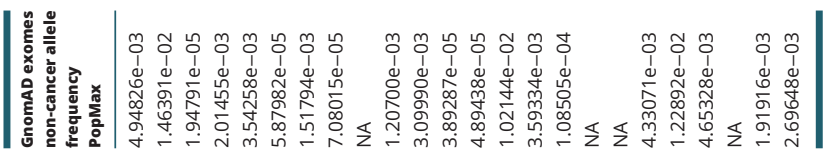

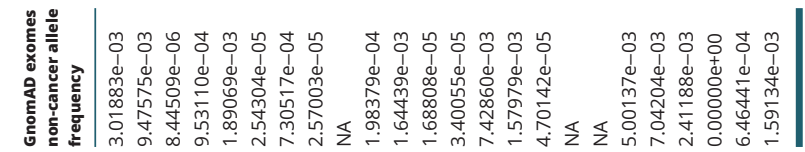

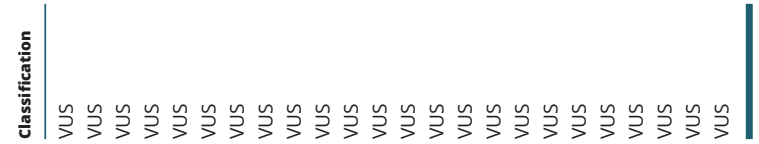

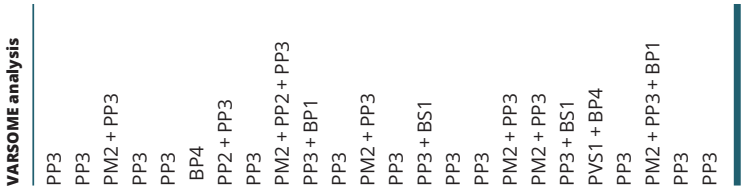

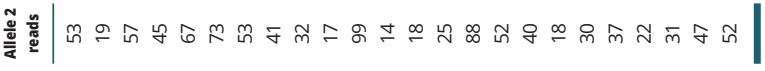

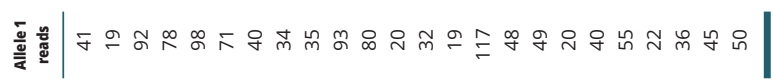

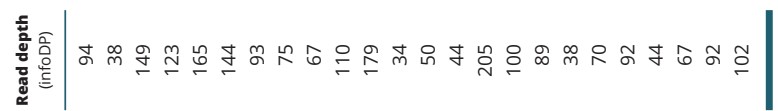
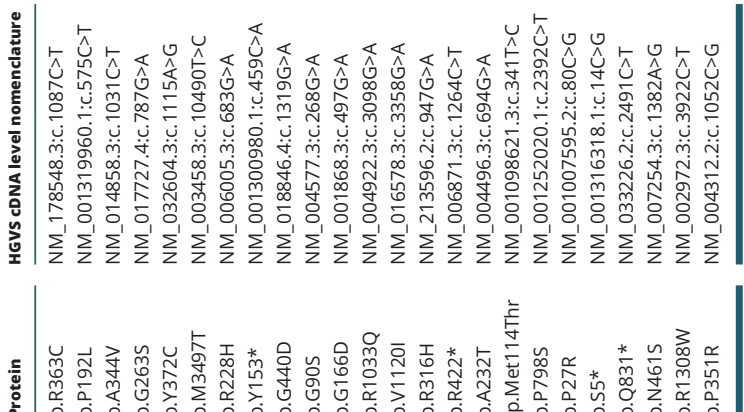

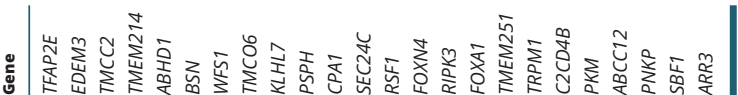
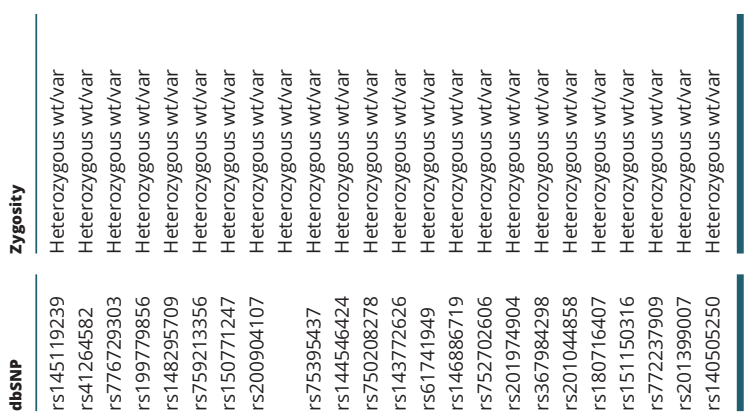

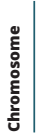

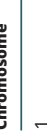

of MAX gene and causality of the p.Ser49Pro variant in the pathogenesis of the PCCs. Thirdly, the introduction of a proline at position 49 seems incompatible with a native 3D conformation for the MAX protein. In addition, although histology of the adrenal nodule identified in patient II-3 is presently not available, its imaging pattern is compatible with a PCC. If confirmed in the follow-up, this diagnosis provides support to the pathogenic role of the MAX variant since II-3 is WT for KIF1B.

The interpretation of the pathogenesis of the PCCs occurring in the proband and her grandfather is more intricate. They both have the MAX p.Ser49Pro plus the KIF1B p.Ser1481Asn germline variants. We were unable to identify LOH of KIF1B (1) or MAX WT alleles in the proband's PCC despite the analyses of multiple separate fragments from the most recently removed PCC which was not fixed in Bouin's reagent known to be deleterious for DNA (21). However, as discussed previously, our additional data on this tumor does not allow us to rule out the contribution of high levels of nontumoral tissue to the lack of detectable LOH. Although we cannot exclude that both KIF1B and MAX may contribute to the PCC phenotype or to the clinical variability in this family, KIF1B pathogenic variants have rarely been described in patients with PCC/ PGL since our initial report in $2008(1,7,8)$. Only one, a p.Tyr835Cys variant, was reported by Welander et al. in a 54-year-1old woman with a unilateral PCC and an endometrial carcinoma (7). This patient had no germline variants in any of the 11 other major susceptibility genes for PCC/PGL but no somatic LOH of the wild-type allele was found, so preventing any definitive conclusion on the pathogenic relevance of this novel variant.

Given the phenotypic variability of this family, with multiple non-PCC/PGL cancers in patient III.1, and to evaluate the possibility that other susceptibility events were at play, we performed WES on the germline DNAs from patient III.1 and II.2. Our working model was that patient III.1 had de novo or maternally-inherited mutations in new/known susceptibility genes for hereditary cancers or, alternatively, in 'modifier genes' which could have an impact on cancer-promoting pathways (24). These additional nucleotide variants, combined with the KIF1B and MAX germline variants, might explain the very severe phenotype of this patient. WES excluded a pathogenic mutation in other genes predisposing to familial PCC/ PGL and also in 30 other hereditary cancer susceptibility genes (22). Among the 125 variants which were private to the proband most were classified as benign or probably benign and were filtered out; the 24 remaining variants were all classified as VUS. Based on bibliographic data,

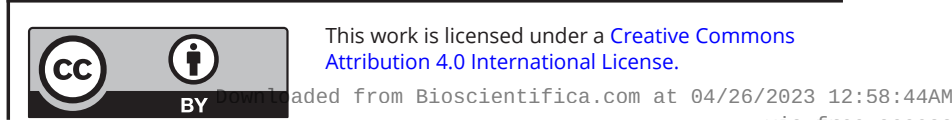


Table 2 Distribution of the five VUS in the germline DNA of the different family members.

\begin{tabular}{l}
\hline Gene \\
\hline Nucleotide variant \\
Protein change \\
Patients \\
III.1 \\
III.2 \\
III.3 \\
II.1 \\
II.2 \\
II.3 \\
I.1
\end{tabular}

\begin{tabular}{l}
\hline TMEM214 \\
\hline c.787G $>A$ \\
p.G263S \\
+ \\
+ \\
Wild type \\
+ \\
Wild type \\
Wild type \\
Wild type
\end{tabular}

\begin{tabular}{l}
\hline KLHL7 \\
\hline C.1319G>A \\
p.G440D \\
+ \\
Wild type \\
+ \\
+ \\
Wild type \\
Wild type \\
Wild type
\end{tabular}

\begin{tabular}{l}
\hline PKM \\
\hline C.14C>G \\
p.S5* \\
+ \\
+ \\
Wild type \\
+ \\
Wild type \\
Wild type \\
Wild type
\end{tabular}

\begin{tabular}{l}
\hline RIPK3 \\
\hline C.1264C>T \\
p.R422* \\
+ \\
+ \\
Wild type \\
+ \\
Wild type \\
Wild type \\
Wild type \\
\hline
\end{tabular}

TFAP2E

C.1087C>T p.R363C

$+$

Wild type

Wild type

Wild type

Wild type Wild type tissue pattern of expression, biological function of the encoded protein, and a catalog of somatic mutations, we narrowed down the list to five variants in five different genes which may be implicated either in the pathogenesis of PCC (KLHL7 and PKM) or sarcomas (RIPK3, TMEM214 and TFAP2E). We did not detect a LOH of the WT allele of KLHL7 or PKM in the proband's PCC. Thus, we cannot assign any causality in the pathogenesis of the proband's PCCs to one of these variants.

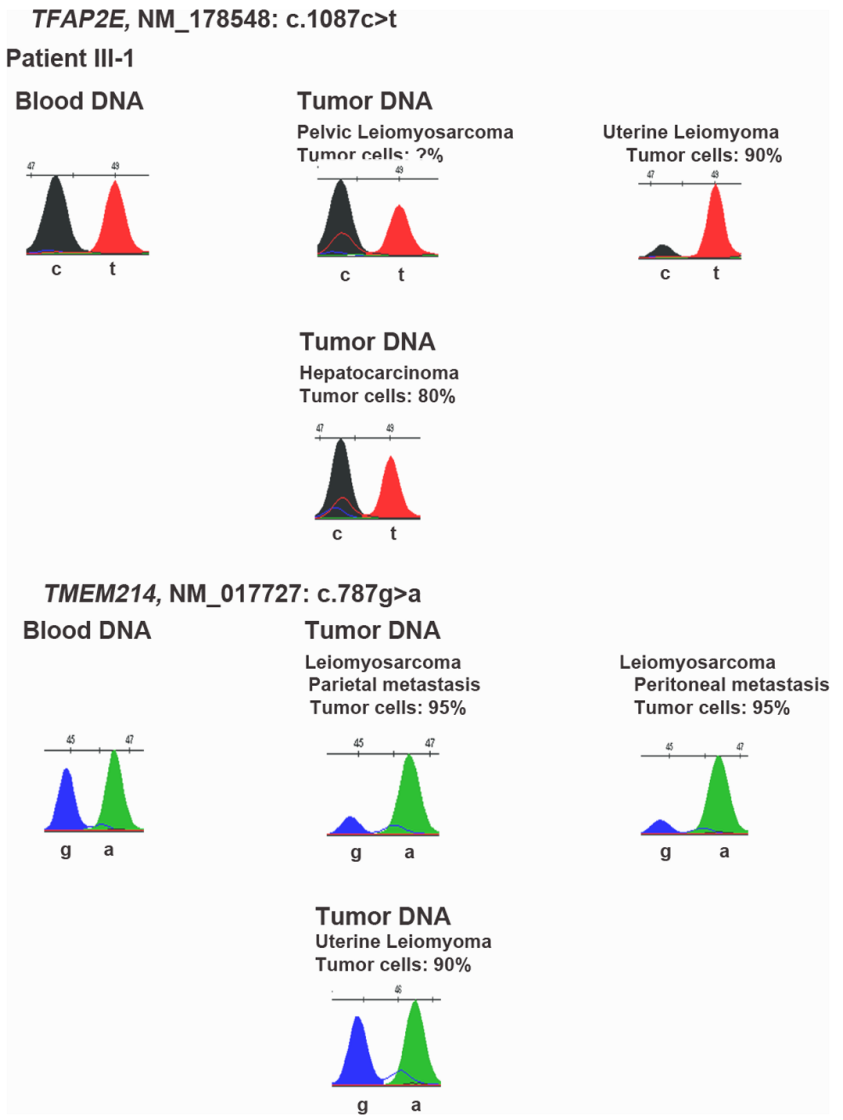

Figure 4

Search for a LOH of TFAP2E and TMEM214 in the DNA extracted from the different tumors from patient III.1 by SnapShot analysis.
We propose that in this family the genetic susceptibility to PCC/PGL is linked to the MAX nucleotide variant which is however associated with an incomplete penetrance since patient II-2 did not develop any symptomatic adrenal lesion. The KIF1B isoform $\beta$ p.Ser1481Asn variant, which is partly defective in the apoptotic culling of neural crest progenitors, may also contribute to the occurrence of NB in the childhood of patient III-1, similar to other rare observations in NB (3). Finally, the leiomyosarcoma and hepatic carcinoma of the proband, and her father's lung adenocarcinoma may be MAX and KIF1B independent. The involvement of TFAP2E and TMEM214 variants is an attractive hypothesis but the pathways acting in disease development remain to be determined.

Supplementary materials

This is linked to the online version of the paper at https://doi.org/10.1530/ EC-20-0460.

\section{Declaration of interest}

The authors declare that there is no conflict of interest that could be perceived as prejudicing the impartiality of the research reported.

\section{Funding}

This work was supported in part by the Association de Recherche en Endocrinologie et Métabolismes (grant to P P).

\section{Acknowledgements}

This work is dedicated to the memory of Pr Charles Proye who was the first to take care of this family and to highlight the hereditary nature of the PCC. We thank Mrs Shahida Flores for her help with data analysis, Dr $\mathrm{N}$ Burnichon and B Bressac de Paillerets for their help with $\mathrm{FH}$ analysis.

\section{References}

1 Yeh IT, Lenci RE, Qin Y, Buddavarapu K, Ligon AH, Leteurtre E, Do Cao C, Cardot-Bauters C, Pigny P \& Dahia PLM. A germline mutation of the $K I F 1 B \beta$ gene on 1 p36 in a family with neural and nonneural

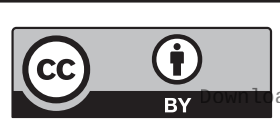

This work is licensed under a Creative Commons Attribution 4.0 International License. 
tumors. Human Genetics 2008124 279-285. (https://doi.org/10.1007/ s00439-008-0553-1)

2 Zhao C, Takita J, Tanaka Y, Setou M, Nakagawa T, Takeda S, Yang HW, Terada S, Nakata T, Takei Y, et al. Charcot-Marie-Tooth disease type $2 \mathrm{~A}$ caused by mutation in a microtubule motor KIF1B $\beta$. Cell 2001105 587-597. (https://doi.org/10.1016/s00928674(01)00363-4)

3 Schlisio S, Kenchappa RS, Vredeveld LCW, George RE, Stewart R, Greulich H, Shahriari K, Nguyen NV, Pigny P, Dahia PL, et al. The kinesin KIF1B $\beta$ acts downstream from EgIN3 to induce apoptosis and is a potential 1p36 tumor suppressor. Genes and Development 200822 884-893. (https://doi.org/10.1101/gad.1648608)

4 Munirajan AK, Ando K, Mukai A, Takahashi M, Suenaga Y, Ohira M, Koda T, Hirota T, Ozaki T \& Nakagawara A. KIF1Bb functions as a haploinsufficient tumor suppressor gene mapped to chromosome 1 p36.2 by inducing apoptotic death. Journal of Biological Chemistry 2008283 24426-24434. (https://doi.org/10.1074/jbc.M802316200)

5 Aarts M, Dannenberg H, de Leeuw RJ, van Nederveen FH, Verhofstad AA, Lenders JW, Dinjens WNM, Speel EJM, Lam WL \& de Krijger RR. Microarray-based CGH of sporadic and syndromerelated pheochromocytomas using a $0.1-0.2 \mathrm{Mb}$ BAC array spanning chromosome arm 1p. Genes, Chromosomes and Cancer 200645 83-93. (https://doi.org/10.1002/gcc.20268)

6 Chen ZX, Wallis K, Fell SM, Sobrado VR, Hemmer MC, Ramskold D, Hellman U, Sansberg R, Kenchappa RS, Martinson T, et al. RNA helicase A is a downstream mediator of KIF1Bb tumor-suppressor function in neuroblastoma. Cancer Discovery 20144 434-451. (https://doi.org/10.1158/2159-8290.CD-13-0362)

7 Welander J, Andreasson A, Christofer Juhlin CC, Wiseman RW, Backdahl M, Hoog A, Larsson C, Gimm O \& Soderkvisst P. Rare germline mutations identified by targeted next-generation sequencing of susceptibility genes in pheochromocytoma and paraganglioma. Journal of Clinical Endocrinology and Metabolism 2014 99 E1352-E1360. (https://doi.org/10.1210/jc.2013-4375)

8 Curras-Freixes M, Pineiro-Yanez E, Montero-Conde C, ApellanizRuiz M, Calsina B, Mancikova V, Remacha L, Richter S, Ercolino T, Rogowski-Lehmann N, et al. PheoSeq : a targeted next-generation sequencing assay for pheochromocytoma and paraganglioma diagnostics. Journal of Molecular Diagnostics 201719 575-588. (https://doi.org/10.1016/j.jmoldx.2017.04.009)

9 Neumann HPH, Young WF \& Eng C. Pheochromocytoma and paraganglioma. New England Journal of Medicine 2019381 552-565. (https://doi.org/10.1056/NEJMra1806651)

10 Toledo RA, Burnichon N, Cascon A, Benn DE, Bayley JP, Welander J, Tops CM, Firth H, Dwight T, Ercolino T, et al. NGSnPPGL study group. Nature Reviews Endocrinology 201713 233-247. (https://doi. org/10.1038/nrendo.2016.185)

$11 \mathrm{Li} \mathrm{H} \&$ Durbin R. Fast and accurate long-read alignment with Burrows Wheeler transform. Bioinformatics 201026 589-595. (https://doi.org/10.1093/bioinformatics/btp698)

12 McKenna A, Hanna M, Banks E, Sivachenko A, Cibulskis K, Kernytsky A, Garimella K, Alsthuler D, Gabriel S, Daly M, et al. The Gonome Analysis ToolKit: a MapReduce Framework for analyzing next-generation DNA sequencing data. Genome Research 201020 1297-1303. (https://doi.org/10.1101/gr.107524.110)

13 Gnirke A, Melnikov A, Maguire J, Rogov P, LeProust EM, Brockman W, Fennell T, Giannoukos G, Fisher S, Russ C, et al. Solution hybrid selection with ultra-long oligonucleotides for massively parallel targeted sequencing. Nature Biotechnology 200927 182-189. (https://doi.org/10.1038/nbt.1523)

14 DePristo MA, Banks E, Poplin R, Garimella KV, Maguire JR, Hartl C, Philippakis AA, del Angel G, Rivas MA, Hanna M, et al. A framework for variation discovery and genotyping using next generation sequencing data. Nature Genetics 201143 491-498. (https://doi. org/10.1038/ng.806)

15 Van der Auwera GA, Carneiro MO, Hartl C, Poplin R Del Angel G Levy-Moonshine A, Jordan T, Shakir K, Roazen D, Thibault J, et al. From FastQ data to high confidence variants calls: the Genome Analysis Tool Kit best practices pipeline. Current Protocols in Bioinformatics 201343 11.10.1-11.10.33. (https://doi. org/10.1002/0471250953.bi1110s43)

16 Kern B, Coppin L, Romanet P, Crépin M, Szuster I, Renaud F, Leteurtre E, Frénois F, Wemeau JL, Carnaille B, et al. Multiple HABP2 variants in familial papillary thyroid carcinoma: contribution of a group of 'thyroid checked' controls. European Journal of Medical Genetics 201760 178-184. (https://doi.org/10.1016/j. ejmg.2017.01.001)

17 Muller M, Guillaud-Bataille M, Salleron J, Genestie C, Deveaux S, Slama A, Bressac de Paillerets B, Richard S, Benusiglio PR \& Ferlicot S. Pattern multiplicity and $\mathrm{FH} / 2$-succino-cysteine staining but not eosinophilic nuclei with perinucleolar halos differentiate hereditary leiomyomatosis and RCC-associated RCC from kidney tumors without FH gene alteration. Modern Pathology 201831 974-983. (https://doi.org/10.1038/s41379-018-0017-7)

18 Maiti R, van Domselaar GH, Zhang H \& Wishart DS. SuperPose: a simple server for sophisticated structural superposition. Nucleic Acids Research 200432 W590-W594. (https://doi.org/10.1093/nar/ gkh477)

19 Kopanos C, Tsiolkas V, Kouris A, Chapple CE, Albarca Aguilera M, Meyer R \& Massouras A. VarSome: the human genomic variant search engine. Bioinformatics 201935 1978-1980. (https://doi. org/10.1093/bioinformatics/bty897)

20 Burnichon N, Cascón A, Schiavi F, Morales NP, Comino-Méndez I, Abermil N, Inglada-Pérez L, de Cubas AA, Amar L, Barontini M, et al. MAX mutations cause hereditary and sporadic pheochromocytoma and paraganglioma. Clinical Cancer Research 201218 2828-2837. (https://doi.org/10.1158/1078-0432.CCR-12-0160)

21 Greer CE, Peterson SL, Kiviat NB \& Manos MM. PCR amplification from paraffin-embedded tissues. Effects of fixative and fixation time. American Journal of Clinical Pathology 199195 117-124. (https://doi. org/10.1093/ajcp/95.2.117)

22 Neben CL, Zimmer AD, Stedden W, van der Akker J, O'Connor R, Chan RC, Chen E, Tan Z, Leon A, Ji J, et al. Multi-gene panel testing of 23,179 individuals for hereditary cancer risk identifies pathogenic variant carriers missed by current genetic testing guidelines. Journal of Molecular Diagnostics 201921 646-657. (https://doi.org/10.1016/j jmoldx.2019.03.001)

23 Uhlén M, Fagerberg L, Hallström BM, Lindskog C, Oksvold P, Mardinoglu A, Sivertsson Å, Kampf C, Sjöstedt E, Asplund A, et al. Proteomics. Tissue-based map of the human proteome. Science 2015 347 1260419. (https://doi.org/10.1126/science.1260419)

24 Ariffin H, Hainaut P, Puzio-Kuter A, Choong SS, Chan AS, Tolkunov D, Rajagopal G, Kang W, Lim LL, Krishnan S, et al. Whole genome sequencing analysis of phenotypic heterogeneity and anticipation in Li-Fraumeni cancer predisposition syndrome. PNAS 2014111 15497-15501. (https://doi.org/10.1073/pnas.1417322111)

Received in final form 17 September 2020

Accepted 8 October 2020

Accepted Manuscript published online 8 October 2020 https://ec.bioscientifica.com https://doi.org/10.1530/EC-20-0460 (c) 2020 The authors Published by Bioscientifica Ltd

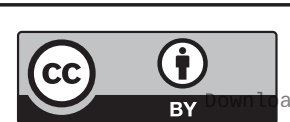

This work is licensed under a Creative Commons Attribution 4.0 International License. ded from Bioscientifica.com at 04/26/2023 12:58:44AM 